\section{THE AMERICAN JOURNAL OF}

\section{PSYCHIATRY}

\section{Contents}

\section{June 2015, Volume 172}

\section{Editorials}

499 Alcohol use disorders: tracts, twins, and trajectories George F. Koob

502 Attention must be paid: the association of plasma clozapine/NDMC ratio with working memory Herbert Y. Meltzer

505 The role of stimulants in late-life depression J. Craig Nelson

508 Parents and genes and their effects on alcohol, drugs, and crime in triparental families Anita Thapar

\section{Introspection}

510 On being chronically ill Mark D. Rego

\section{Clinical case conference}

512 Psychiatric care during hepatitis $\mathrm{C}$ treatment: the changing role of psychiatrists in the era of direct-acting antivirals Sanjeev Sockalingam, Kathleen Sheehan, Jordan J. Feld Hemant Shah

\section{Images in psychiatry}

517 The stone that mourns its victims: Haiti still recovering from its injuries and traumas 5 years after the 2010 earthquake

Jude Mary Cénat, Patricia Eid, Daniel Derivois, Martine Hébert, Lewis Ampidu Clorméus

\section{Reviews and overviews}

519 CBT for adolescents with anxiety: mature yet still developing

Philip C. Kendall, Jeremy S. Peterman

\section{New research - articles}

531 Brain development in heavy-drinking adolescents Lindsay M. Squeglia, Susan F. Tapert, Edith V. Sullivan, Joanna Jacobus, M. J. Meloy, Torsten Rohlfing, Adolf Pfefferbaum

543 Association of protein phosphatase PPM1G with alcohol use disorder and brain activity during behavioral control in a genome-wide methylation analysis Barbara Ruggeri, Charlotte Nymberg, Eero Vuoksimaa, Anbarasu Lourdusamy, Cybele P. Wong, Fabiana M. Carvalho, Tianye Jia, Anna Cattrell, Christine Macare, Tobias Banaschewski Gareth J. Barker, Arun L.W. Bokde, Uli Bromberg, Christian Büchel, Patricia J. Conrod, Mira Fauth-Bühler, Herta Flor, Vincent Frouin, Jürgen Gallinat, Hugh Garavan, Penny Gowland, Andreas Heinz, Bernd Ittermann, Jean-Luc Martinot, Frauke Nees, Zdenka Pausova, Tomá Paus, Marcella Rietschel, Trevor Robbins, Michael N. Smolka, Rainer Spanagel, Georgy Bakalkin Jonathan Mill, Wolfgang H. Sommer, Richard J. Rose, Jia Yan, Fazil Aliev, Danielle Dick, Jaakko Kaprio, Sylvane Desrivières, Gunter Schumann, the IMAGEN Consortium

553 Triparental families: a new genetic-epidemiological design applied to drug abuse, alcohol use disorders, and crimina behavior in a Swedish national sample Kenneth S. Kendler, Henrik Ohlsson, Jan Sundquist, Kristina Sundquist

561 Citalopram, methylphenidate, or their combination in geriatric depression: a randomized, double-blind placebo-controlled trial

Helen Lavretsky, Michelle Reinlieb, Natalie St. Cyr, Prabha Siddarth, Linda M. Ercoli, Damla Senturk

570 Cerebral small vessel disease and association with higher incidence of depressive symptoms in a general elderly population: the AGES-Reykjavik study

Thomas T. van Sloten, Sigurdur Sigurdsson, Mark A. van Buchem, Caroline L. Phillips, Palmi V. Jonsson, Jie Ding, Miranda T. Schram, Tamara B. Harris, Vilmundur Gudnason, Lenore J. Launer

579 Prediction of working memory performance in schizophrenia by plasma ratio of clozapine to $\mathrm{N}$-desmethylclozapine Tarek K. Rajji, Benoit H. Mulsant, Simon Davies, Sawsan M. Kalache, Christopher Tsoutsoulas, Bruce G. Pollock, Gary Remington 